\title{
FRANCISCO PACHECO Y EL RETRATO DE MIGUEL DE CERVANTES. UN ANÁLISIS A TRAVÉS DE LA PRENSA SEVILLANA DEL SIGLO XIX
}

\author{
FRANCISCO PACHECO AND THE PORTRAIT OF MIGUEL \\ DE CERVANTES. AN ANALYSIS THROUGH SEVILLIAN \\ NINETEENTH CENTURY PRESS
}

\author{
Carmen Rodríguez Serrano \\ Universidad de Sevilla, España \\ rodriguezserranocarmen@gmail.com
}

Este estudio tiene la intención de dar a conocer la efigie cervantiva más representada, extraída de un posible retrato incluido dentro de la pintura San Pedro Nolasco redimiendo a los cautivos, ejecutada por Francisco Pacheco a finales del siglo XVI. Esta labor se llevará a cabo a través del análisis de la literatura artística que se desarrolla en Sevilla durante el siglo XIX y en concreto de El Ateneo, Periódico de Literatura Española y Extranjera, Ciencias y Bellas Artes, publicación que gracias a la figura de su director, José María Asensio y a los artículos de Antoine de Latour, será una fuente muy valiosa de información para el estudio del verdadero retrato del autor del Quijote.

Palabras claves: Retrato, prensa sevillana del siglo XIX, Francisco Pacheco, Miguel de Cervantes.

This study intends to show an image of the most popular personification of Cervantes, from a possible portrait shown in San Pedro Nolasco redeeming captives, painted by Francisco Pacheco. This paper will analyze his figure through the artistic literature developed in Seville during the 19th century and through El Ateneo, Periódico de Literatura Española y Extranjera, Ciencias y Bellas Artes - very valuable source of information for the study of the real portrait of the author of El Quijote. This was possible thanks to his director, Jose Maria Asensio and Antoine de Latour's articles.

Keywords: Portrait, Sevillian Nineteenth Century Press, Francisco Pacheco, Miguel de Cervantes.

El conocimiento del aspecto físico del escritor más importante de la cultura hispánica, Miguel de Cervantes, despertó, desde muy pronto, el interés de la intelectualidad española y europea. Durante el siglo XVIII se realizaron algunas representaciones del mismo, aunque será el siglo XIX el que con mayor frecuencia 
lo reproduzca y plantee el debate sobre la veracidad en su retrato. Fueron diversas las fuentes que sirvieron para ilustrar estas obras, aunque hay que señalar que se consideraron escasas y poco fiables. Entre ellas siempre se tuvieron en cuenta los retratos que realizaron dos artistas coetáneos al pintor, Francisco Pacheco y Juan de Jáuregui, los cuales se hallaban prácticamente perdidos, conservándose tan solo alguna mala copia que distorsionaba al original. El deseo por conocer el rostro del escritor, como ya se apuntó, llevó a parte de los intelectuales del siglo XVIII a investigar al respecto, realizándose alguna representación de Cervantes, aunque de dudosa veracidad, caso del retrato que ilustró la edición inglesa del Quijote en 1738 o el que se realizó en 1780 para la Academia Española, a través de un óleo propiedad del Conde del Águila en Sevilla. Ambos retratos se basaban en un mismo modelo erróneo o al menos así será señalado durante el siglo XIX. Éste trajo nuevas investigaciones que buscaban aclarar el aspecto de Cervantes, como las realizadas por José María Asensio, estudioso cervantino y de la figura de Francisco Pacheco, que según la opinión de la intelectualidad nacional de la época, realizó un hallazgo significativo, no obstante hoy, es puesto en tela de juicio.

Durante la centuria decimonónica fueron varias las ciudades españolas que intentaron relacionarse de algún modo con la figura de Cervantes, siendo la literatura artística sevillana una de las más interesadas. No hay que olvidar que la mentalidad romántica, tan arraigada en muchos aspectos a la ciudad de Sevilla, motivará la búsqueda de elementos que les hagan sentirse orgullosos de su historia. En esta línea, el presente artículo aborda el estudio llevado a cabo por Asensio, que determinaba la relación entre una obra de Francisco Pacheco y el verdadero retrato de Miguel de Cervantes, pero desde el análisis realizado a través de una publicación sevillana de la segunda mitad del siglo XIX. El estudioso de Pacheco será además, el director de El Ateneo Periódico de Literatura Española y Extranjera, Ciencias y Bellas Artes ${ }^{1}$, que estuvo vigente en Sevilla desde su fundación el 1 de diciembre de 1874 hasta su desaparición el 15 de noviembre de 1875 , manteniendo una periodicidad quincenal ${ }^{2}$. Asensio fue una de las personalidades más destacadas dentro del panorama cultural hispalense y nacional, circunstancia que benefició desde un primer momento a esta publicación, ya que incentivó la participación activa de la élite intelectual, reunida en torno a su figura. Así, fueron muchos los ilustres redactores y colaboradores, todos ellos vinculados de uno u otro modo con la ciudad de Sevilla, los que realizaron sugestivos

${ }^{1}$ El Ateneo, Periódico de Literatura española y extranjera, Ciencias y Bellas Artes, Sevilla, Tipográfica Francisco Álvarez y Compañía, editores de Cámara de S.M, 1874-1875.

${ }^{2}$ El ejemplar consultado procede de la donación Montoto a la biblioteca de la Universidad de Sevilla. Los veinticuatro volúmenes se encuentran encuadernados en un mismo tomo, lo que ha facilitado su consulta. Se puede encontrar también en su completa totalidad en la Hemeroteca Municipal de Sevilla. 
trabajos sobre crítica, historia y bibliografía española, entre ellos Joaquín Guichot y Parody, Antoine de Latour, Enriqueta Madoz de Aliana, Ramón de Campoamor, Manuel Cano y Cueto, Antonia Díaz de Lamarque, Luis Montoto o Cecilia Böhl de Faber ${ }^{3}$. Como no, Asensio será uno de los más activos colaboradores en el periódico, realizando una gran cantidad de artículos con tintes artístico-literarios, en gran parte dedicados a la figura de Cervantes y Pacheco, tal y como ya se indicó.

\section{EL VERDADERO RETRATO DE CERVANTES}

La curiosidad por conocer la fisonomía del autor del Quijote, siempre estuvo presente, aunque no eran abundantes las fuentes documentales y mucho menos las artísticas. Muy significativa e ilustrativa resulta en este sentido, la descripción que hizo de sí mismo el propio Cervantes en el prólogo de las Novelas Ejemplares ${ }^{4}$, clave para entender la relación que establecerá posteriormente José María Asensio con la obra de Pacheco:

“(...) Este que veis aquí del rostro aguileño, de cabello castaño, frente lisa y desembarazada, de alegres ojos y de nariz corba, aunque bien proporcionada, las barbas de plata, que no há veinte años, que fueron de oro, los bigotes grandes, la boca pequeña, los dientes no crecidos porque no tiene sino seis, y esos mal acondicionados y peor puestos porque no tienen correspondencia los unos con los otros, el cuerpo entre dos extremos, ni grande ni pequeño, la color viva, antes blanca que morena, algo cargado de espaldas, y no muy ligero de pies: este digo que es el rostro del autor de la Galatea y de Don Quijote de la Mancha, y el que hizo el Viaje del Parnaso a imitación del de Cesar Caporal Perusino y otras obras que andan por ahí descarriadas, y quizás sin el nombre de su dueño: llámase comúnmente Miguel de Cervantes Saavedra (...) $)^{5}$.

La autodescripción de Cervantes va a dar lugar a diversos estudios artísticos que vinculen ciertos retratos con la efigie del propio escritor, caso de la investigación llevada a cabo por Asensio en 1864, que no tendrá una fuerte repercusión hasta no ser presentada por la prensa artística de la segunda mitad del siglo XIX. Con motivo del CCLIX aniversario de la muerte de Cervantes, el 23 de abril de $1875^{6}$, El Ateneo publicó un número especial que contenía un interesante artículo de Antoine de Latour, sobre los retratos de Cervantes. Ésta era la primera parte de la publicación, que fue completada el día 1 de mayo, cuando salió a la luz $\mathrm{N}^{0} 11^{7}$.

${ }^{3}$ CHAVES REY, Manuel: Historia y Bibliografia de la Prensa Sevillana, Sevilla, Imprenta de E. Rasco, 1896, pp. 205-206.

${ }^{4}$ CERVANTES SAAVEDRA, Miguel de: Novelas ejemplares, Madrid, Cátedra, 1995.

${ }^{5}$ CERVANTES SAAVEDRA, Miguel de: op. cit.

${ }^{6}$ El Ateneo..., op. cit., pp. 126-128.

${ }^{7}$ Ibídem, pp. 141-142. 
La importancia de estos escritos reside en la defensa de la teoría que apunta al hallazgo por parte de Asensio del verdadero modelo del retrato que Francisco Pacheco realizó de Miguel de Cervantes a finales del siglo XVI y que se creía perdido. Éste se encuentra inserto dentro de otra pintura, y en él, el escritor es caracterizado como un personaje anónimo. Se va a establecer así, una relación entre uno de los integrantes de la composición San Pedro Nolasco redimiendo a los cautivos (Figuras 1 y 2) y los rasgos faciales característicos en el manco de Lepanto. La pintura es de manera independiente a su iconografía religiosa, que muestra un episodio de la vida del santo, un retrato colectivo de la época. La escena presenta a San Pedro Nolasco en primer plano junto a un cautivo que se dispone a coger al santo para llevarlo a una barca representada en segundo plano. En ella, hay otro religioso junto a otros dos cautivos que sujetan un cofre, con el escudo de la Orden de la Merced. Como no, aparece un barquero que se gira bruscamente hacia el espectador, buscando ser visto y que según Asensio, es el personaje elegido para presentar a Cervantes. No es de extrañar que fuese El Ateneo la publicación donde se pusiera en valor la figura de Pacheco y donde se defendiera la veracidad en el posible retrato de Cervantes. Este artículo, aparece a raíz de un texto realizado por el propio Asensio en 1864, como ya se señaló, Nuevos documentos para ilustrar la vida de Miguel de Cervantes Saavedra; con algunas observaciones y artículos sobre la vida y obra del mismo autor, y las pruebas de la autenticidad de su verdadero retrato ${ }^{8}$, donde expone esta teoría. El texto, de gran interés, recoge además un dibujo realizado por Eduardo Cano, en el cual se reproduce el detalle en medio cuerpo, del barquero de Pacheco, siendo por tanto este el primero de los ejemplos gráficos que lo vincula. En él, Asensio presenta una serie de tesis con las que avala la autenticidad del retrato de Cervantes y señala como hasta ese momento, los retratos que habían recogido la efigie del literato, estaban faltos de autenticidad. Para él este sí es veraz, apoyándose para su tesis, en intelectuales como Joaquín Domínguez Bécquer y Eduardo Cano, pintores profesores de la escuela de Sevilla, además de seguidores de la obra de Cervantes, que confirmarán que la obra de Pacheco es un retrato colectivo de la época en la que fue realizado.

A pesar de la investigación de Asensio, será De Latour el que realmente dé difusión a la noticia entre la intelectualidad sevillana y subraye su importancia, señalando que "Solo se conocía de él un retrato de dudosa autenticidad; pero acaba de descubrirse otro que parece reunir todos los caracteres de verdadero ${ }^{9}$ "y apoyando firmemente la afirmación, búsqueda y hallazgo del director de El Ateneo:

${ }^{8}$ ASENSIO, José María: Nuevos documentos para ilustrar la vida de Miguel de Cervantes Saavedra; con algunas observaciones y articulos sobre la vida y obra del mismo autor, y las pruebas de la autenticidad de su verdadero retrato, Sevilla, Imprenta y Litografía Librería Española y Extrangera, 1864.

${ }^{9}$ El Ateneo..., op. cit., p.126. 
“(...) El verdadero descubridor es el que busca, y sabe lo que busca, y por qué lo busca. Hace muchos años que el Sr. Asensio perseguía, por decirlo así, el retrato de Cervantes. Creía firmísimamente en su existencia lo adivinaba por el poder de su convicción. Sabía que Pacheco, contemporáneo y amigo del autor de D. Quijote, había reproducido aquella figura ilustre, y Sevilla guarda aún en su Museo y sus iglesias gran número de lienzos de aquel maestro. ¿En cuál de aquellos cuadros se encontraba aquel importante secreto? (... $)^{10 "}$.

El autor del artículo, siguiendo la teoría de Asensio, señalará que Cervantes estuvo en el taller del suegro de Velázquez, y que sin duda alguna, este lo retrató en su Libro de Descripción de Verdaderos Retratos de Ilustres y Memorables Varo$n e s^{11}$, que recogía las vidas y los retratos de personalidades destacadas, pero que por desgracia se encontraba incompleto ${ }^{12}$, sin poder concluir en los rasgos que identificaban al autor del Quijote. No obstante, lo más interesante son las circunstancias que hicieron posible la relación de Asensio con cierta obra de Pacheco, expuestas por De Latour del siguiente modo:

“(...) Registrando antiguos papeles, el Sr Asensio tropezó un día con cierto manuscrito intitulado "Relación de cosas de Sevilla de 1550 a 1640", relación que tenía carácter de respetable y auténtica. Entre muchos pormenores del más alto interés se leía en ella que Francisco Pacheco y Alonso Vázquez habian pintado en competencia seis grandes lienzos destinados a los claustros del Convento de la Merced, y que en uno de ellos que representaba a los Padres de la Redención, con algunos cautivos a quienes habían rescatado, se encontraba el retrato de Cervantes, y los de otras personas que habían estado en Argel.

Ahora bien, es hoy el Museo de Sevilla, y entre los cuadros que en él se custodian están casualmente los seis lienzos; pero ¿cómo encontrar a Cervantes? A primera vista no se le reconocía en ninguna de las figuras. Pero una dicha nunca viene sola. En la primavera de 1850, fue cuando el señor Asensio recogió esa luminosa indicación. En el mes de mayo de $1864^{13}$ (¿otra primavera afortunada?), logró por fin tras años de inútiles investigaciones, descubrir el mensaje de Pacheco $(. . .)^{14 "}$.

Este hallazgo, unido a la propia descripción que hizo de sí mismo Cervantes, van a ser otra de las claves para que Asensio, identifique al barquero de Pacheco,

${ }^{10}$ Ibídem, p. 126.

${ }^{11}$ PACHECO, Francisco: Libro de Descripción de Verdaderos Retratos de Ilustres y Memorables Varones, Diputación Provincial de Sevilla, Sevilla, 1985.

${ }^{12}$ Esta obra solo conserva algunos retratos, perdiéndose gran número, entre ellos el de Cervantes.

${ }^{13}$ Esta puede ser la fecha en la que Asensio adquirió el Libro de Descripción de Verdaderos Retratos de Ilustres y Memorables Varones de Pacheco, la cual fue de gran ayuda para su investigación.

${ }^{14}$ Ibídem, p. 127. 
con el propio autor del Quijote. De Latour apoya la teoría describiendo al personaje y concluyendo en lo siguiente:

“(...) un trage grosero, un coleto de ante, un sombrero blanco de fieltro, todo el equipo severo e un soldado. ¿Y qué era Cervantes cuando los corsarios le cautivaron? (...) los cabellos son naturalmente rizados; como en el prólogo de las novelas la boca es pequeña, el bigote grande, los ojos vivos, la tez casi blanca, la nariz aguileña pero bien proporcionada, la barba es rubia lo mismo que el bigote. Eran de plata en la época en que Pacheco conoció a Cervantes; pero eran de oro en el momento a que se refiere el pintor, que es el del rescate del escritor. La objeción aquí sería una prueba más en apoyo de la tesis del Sr. Asensio (... $)^{15, "}$

Todas estas pruebas presentadas por José María Asensio y secundadas por Antoine De Latour, van a tener una amplia repercusión en el mundo cervantino, ya que van a ser tomadas por verdaderas, escogiéndose durante el siglo XIX el modelo del barquero como el retrato de Cervantes. Lógicamente, a partir de ese momento, serán numerosas las publicaciones de la época que traten temas dedicados al escritor, las que aparezcan ilustradas con este motivo. Un ejemplo de lo reseñado serán dos grabados presentados en el periódico madrileño, La Ilustración de Madrid ${ }^{16}$ (Figuras 3 y 4), que explican y corroboran un artículo en el que también se apoya la teoría de Asensio, con fecha de 15 de abril de 1872; así como la portada del propio Ateneo, que el 23 de abril de 1875 recogió la efigie de nuestro literato más universal en homenaje a su figura y a la propia mano de Pacheco (Figura 5).

Hay que concluir señalando como la investigación realizada por Asensio resulta muy interesante desde el punto de vista comparativo y sin duda es una base para el estudio histórico-artístico nacional. Del mismo modo, los artículos De Latour, suponen una destacada muestra de la temática abordada por la prensa durante el periodo de la Restauración Borbónica en España. No obstante, no resultan fuentes absolutamente fiables, ya que la investigación actual suele ser más cauta y se basa en pruebas mucho más concretas. No se puede saber con certeza sí ese era el verdadero retrato de Cervantes, ya que incluso si así fuera, Pacheco lo habría pintado desde una perspectiva idealizadora, no objetiva, muy diferente al tipo de retrato que posteriormente realizará su yerno, Diego Velázquez.

Fecha de recepción: 30 de septiembre de 2014

Fecha de aceptación: 28 de noviembre de 2014

\footnotetext{
${ }^{15}$ Ibid, p. 128 .

${ }^{16}$ La Ilustración de Madrid, Madrid, Imprenta de El Imparcial, 1870-1872.
} 


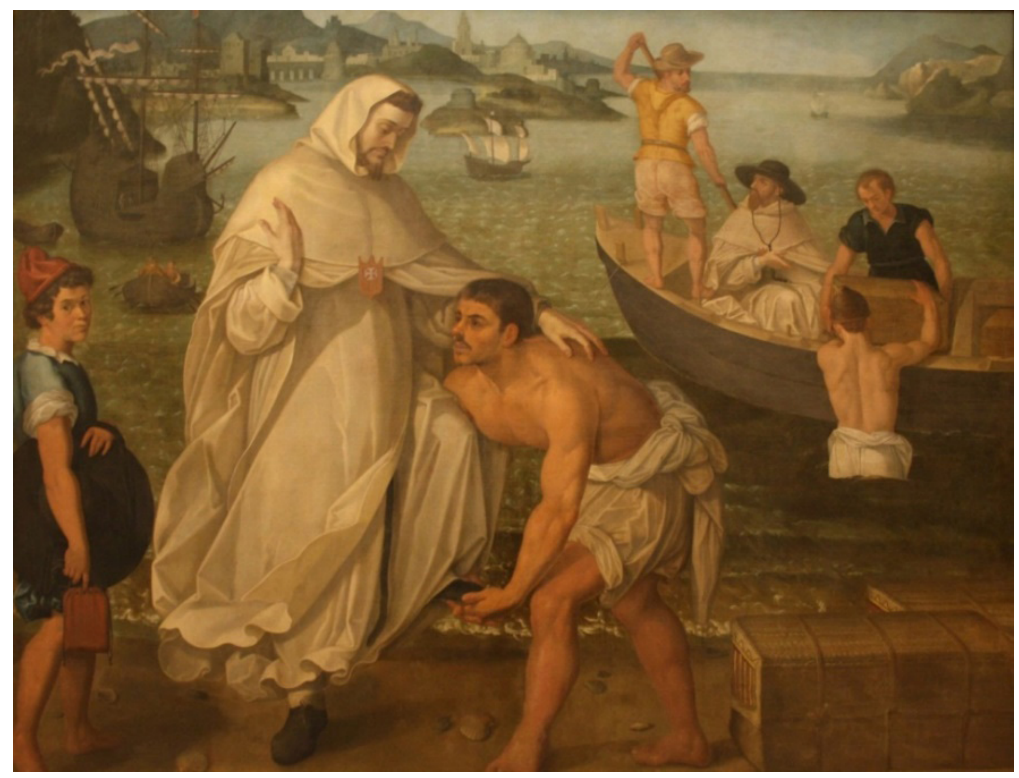

Figura 1. San Pedro Nolasco redimiendo a los cautivos. Francisco Pacheco. 1600-1611. Museo de Bellas Artes de Sevilla.

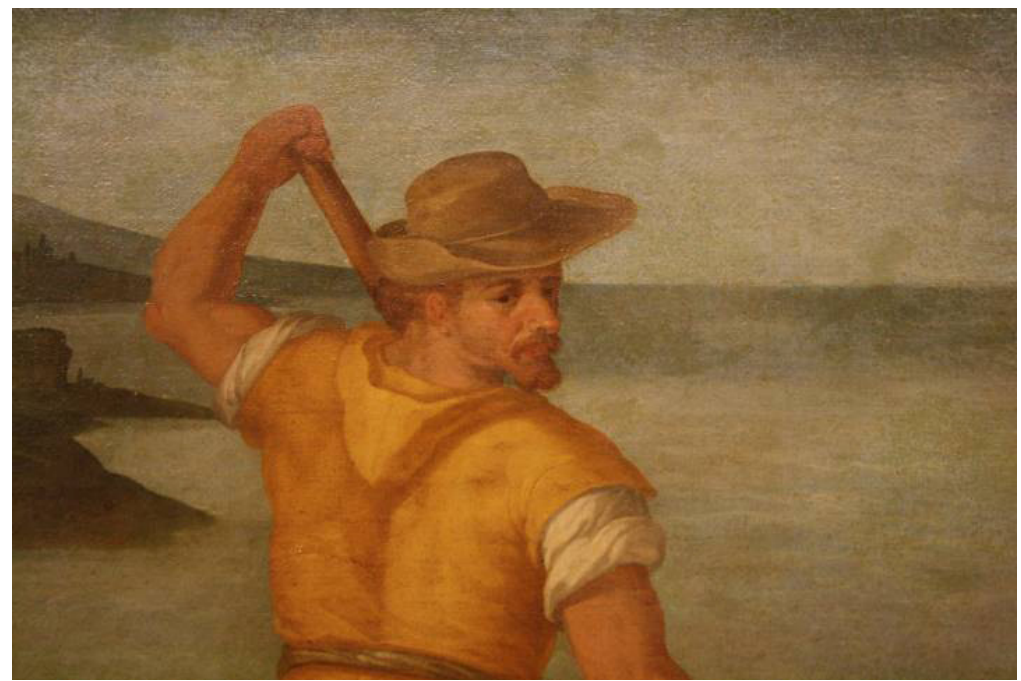

Figura 2. Detalle del barquero en San Pedro Nolasco redimiendo a los cautivos. Francisco Pacheco. 1600-1611. Museo de Bellas Artes de Sevilla. 


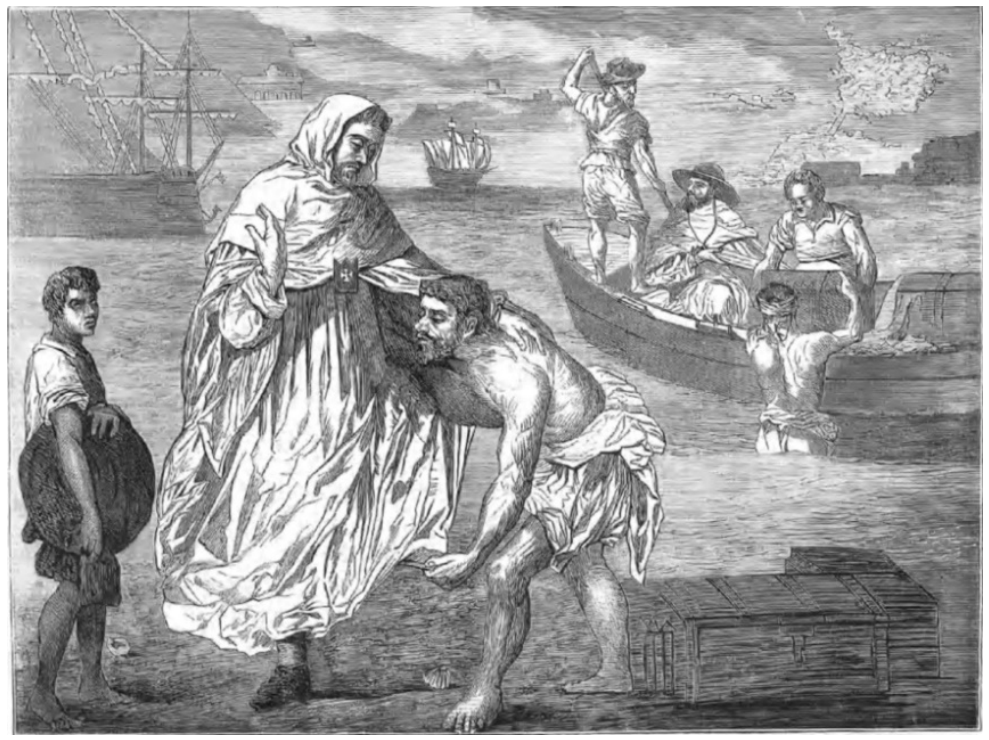

Figura 3. San Pedro Nolasco redimiendo a los cautivos. Grabado sobre pintura de Francisco Pacheco. 1872. La Ilustración de Madrid.

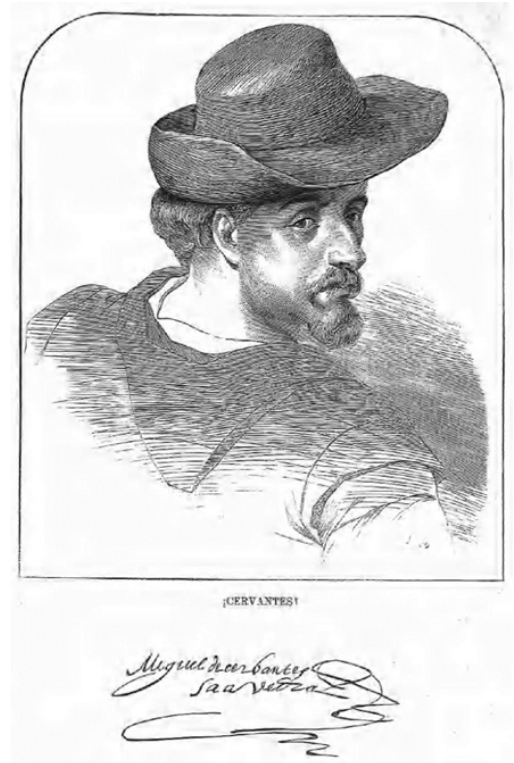

Figura 4. Retrato de Miguel de Cervantes. Grabado. 1872. La Ilustración de Madrid. 


\section{EL ATENEO.}

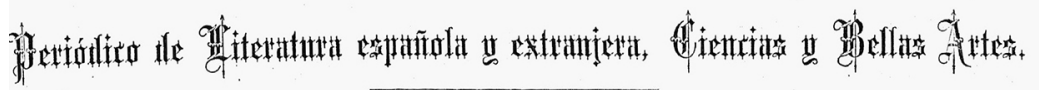

N. 10. VIERNES 23 DE ABRIL 1875.

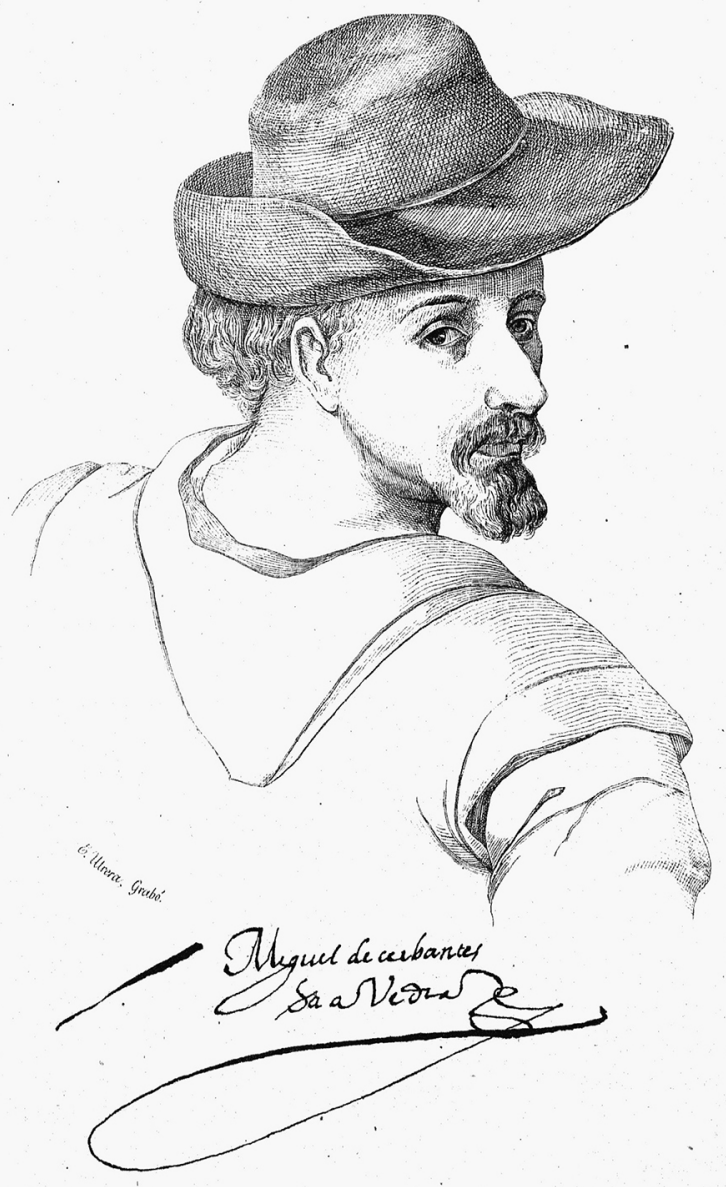

Figura 5. Portada de El Ateneo. 1875. El Ateneo, Periódico de Literatura española y extranjera, Ciencias y Bellas Artes. 